\title{
Proper motion separation of Be star candidates in the Magellanic Clouds and the Milky Way
}

\author{
Katherine Vieira, ${ }^{1 \star}$ Alejandro García-Varela ${ }^{2}$ and Beatriz Sabogal ${ }^{2}$ \\ ${ }^{1}$ Centro de Investigaciones de Astronomía, Apartado Postal 264, Merida 5101-A, Venezuela \\ ${ }^{2}$ Universidad de los Andes, Departamento de Física, Cra. 1 \#18A-10, Bloque Ip, AA 4976, Bogotá, Colombia
}

Accepted 2017 April 26. Received 2017 April 26; in original form 2017 March 20

\begin{abstract}
We present a proper motion investigation of a sample of Be star candidates towards the Magellanic Clouds, which has resulted in the identification of separate populations, in the Galactic foreground and in the Magellanic background. Be stars are broadly speaking B-type stars that have shown emission lines in their spectra. In this work, we studied a sample of 2446 and 1019 Be star candidates towards the LMC and SMC respectively, taken from the literature and proposed as possible Be stars due to their variability behaviour in the OGLE-II I band. JHKs magnitudes from the IRSF catalog and proper motions from the SPM4 catalog, were obtained for 1188 and 619 LMC and SMC Be stars candidates, respectively. Color-color and vector-point diagrams were used to identify different populations among the Be star candidates. In the LMC sample, two populations with distinctive infrared colours and kinematics were found, the bluer sample is consistent with being in the LMC and the redder one with belonging to the Milky Way disk. This settles the nature of the redder sample which had been described in previous publications as a possible unknown subclass of stars among the Be candidates in the LMC. In the SMC sample, a similar but less evident result was obtained, since this apparent unknown subclass was not seen in this galaxy. We confirm that in the selection of Be stars by their variability, although generally successful, there is a higher risk of contamination by Milky Way objects towards redder $\mathrm{B}-\mathrm{V}$ and $\mathrm{V}-\mathrm{I}$ colors.
\end{abstract}

Key words: proper motion - Be stars - Magellanic Clouds

\section{INTRODUCTION}

Be stars are broadly defined as non-supergiant (luminosity class III to V) B-type stars that have or have had Balmer emission lines (Collins 1987). The presence of a flattened circumstellar gaseous disk formed of material ejected from the star, a dust-free Keplerian decretion disc, is currently the accepted explanation for some of the observed features in Be stars: the UV stellar light is reprocessed in it and produces the emission lines, and the observed IR excess and polarization result from the scattering of the stellar light by the disk (see Rivinius et al. (2013) for details). Several mechanisms have been proposed for the mass-ejection process that forms the disk, which are well constrained but not totally understood. In the so-called Classical Be it clearly comes from the rapid rotation of the star, probably along with other processes including non-radial pulsations and smallscale magnetic fields; and in binaries stars the material is

^ E-mail: kvieira@cida.gob.ve being accreted by the companion of the Be star, generally a white dwarf.

Mennickent et al. (2002) and Sabogal et al. (2005), have identified a large number of Be star candidates towards the Magellanic Clouds based on their photometric variability in the OGLE-II I band. Mennickent et al. (2002) has proposed a classification of these variability-selected stars into four types according to their light curves morphology and suggest that Type- 1 and Type- 2 stars could be Be stars with accreting white dwarfs in a Be + WD binary or blue premain-sequence stars showing accretion disc thermal instabilities, Type- 4 stars could be single Be stars and Type- 3 stars should not be linked to the Be star phenomenon at all. They found also a sample of transition stars between Type1 and Type- 2 stars, and suggested that these stars could be experiencing the same phenomenon, which could be outbursts of plasma, accretion from a white dwarf companion, or accretion disc thermal instabilities like these found in blue pre-main sequence stars. This last hypothesis was discarded in the study by Mennickent et al. (2009). 
Paul et al. (2012) showed that the photometric method used in the aforementioned works is effective in the selection of Be star candidates. Their spectroscopic analysis found that most of the stars studied from a sample of such candidates in both LMC and SMC, belong to early type stars with emission supporting circumstellar material. However an enigmatic result was obtained in their work among Type- 4 LMC Be candidate stars: a subgroup of the brightest and most massive stars was found in the sample with no NIR excess and large reddening although they were not located in regions with high reddening. Stars with similar characteristics were not found in the SMC or in our Galaxy and it was proposed they formed a possible subclass of stars that needed further analysis.

In this work we used the Southern Proper Motion 4 catalogue (SPM4) (Girard et al. 2010) to study the kinematics of Be star candidates of the LMC and SMC galaxies, to probe the presence of contaminants or various populations in the studied samples, and with such information gain a better insight into the nature of these stars. This also allowed us to independently evaluate how successful the photometric variability techniques used by Mennickent et al. (2002) and Sabogal et al. (2005) are to select Be star candidates, and confirm that some biases could be present in such method.

\section{CROSS-MATCHING BE STARS WITH IRSF AND SPM4}

Be star candidates for the LMC and SMC were obtained from Sabogal et al. (2005) and Mennickent et al. (2002), respectively. A total of 2446 and 1019 candidates were listed, but we found four and two repeated entries in each catalog. In the LMC, the four sources that appeared twice each, had the same sky coordinates, and very similar magnitudes and colors, only differing in their Type. In each case, one entry was a Type-4, and for the lack of better information, we kept that entry in the catalog and deleted the other. In the SMC, a similar situation was faced and since in the two cases one entry was a Type- 1 star, this one was selected in each case. The final lists of unique entries of Be star candidates to match contained 2442 and 1017 stars in the LMC and SMC, respectively.

A first match by sky coordinates was done between the Be star candidates and the SPM4 catalog, with 3" as a matching radius. A few multiple matches (two Be stars matched to the same SPM4 star and viceversa), were found. Since SPM4 measured a V magnitude, a comparison with their OGLE-II's V could provide additional information to clarify these problematic matches and also to discard false matches, but the SPM4 photometry in the LMC and SMC areas comes mostly from photographic plates and has a poor quality compared to CCD photometry. We also noticed the J-H vs. H-K color-color diagram done with the JHKs 2MASS magnitudes listed by SPM4, suffered from a noticeable higher dispersion, as compared to those published by Paul et al. (2012) using the InfraRed Survey Facility (IRSF) magnitudes by Kato et al. (2007).

Therefore, we decided to make a cross-match between SPM4 and IRSF in the areas that cover the location of the Be star candidates in the LMC and SMC, respectively, to obtain a SPM4 $\times$ IRSF catalog with proper motions and good infrared magnitudes. Then a second match between the Be star candidates and this SPM4 $\times$ IRSF catalog was performed to make the final cross-identifications. While the IRSF photometry is of better quality, the catalog contains a non-negligible portion of false or poor quality entries (extended/faint/saturated/etc. sources), therefore in an effort to consider only good quality point sources, we selected only those with the quality and periphery flags both equal to 111 and the adjacency flag equals to 000. By applying such restrictions, the IRSF catalog was reduced to about $15 \%$ of the original total number of listed entries. To our surprise, we found a few tens of these point sources with repeated IDs, that had the same sky coordinates and similar photometry (within 0.1-0.2 magnitudes), and for the lack of better information, we chose the first one to appear for each case.

As IRSF has a fainter limiting magnitude than SPM4, the risk for mismatches between the catalogs by using only the position in the sky cannot be ignored. But SPM4 2MASS-measured JHKs magnitudes help us to overcome this trouble, by looking also for a similarity in the J-band photometry. We first transformed the 2MASS J magnitude listed in SPM4 to the IRSF system by applying the corresponding transformation, listed in Table 10 of Kato et al. (2007) as $\mathrm{J}_{\text {IRSF }}=\mathrm{J}_{2 \mathrm{MASS}}-0.043\left(\mathrm{~J}_{2 \mathrm{MASS}}-\mathrm{H}_{2 \mathrm{MASS}}\right)+0.018$. Then, for all IRSF sources within 3" in the sky and within 0.5 magnitudes in J magnitude from a SPM4 one, a match is done with the IRSF star with the smallest

$d=\sqrt{\left(\frac{\Delta \theta}{3 »}\right)^{2}+\left(\frac{\Delta \mathrm{J}}{0.5 m a g}\right)^{2}}$

where $\Delta \theta$ is the sky angular separation in arcseconds between the $(\mathrm{ra}, \mathrm{dec})$ coordinates listed in SPM4 and IRSF, and $\Delta \mathrm{J}$ is the difference between the IRSF $\mathrm{J}$ magnitude and the 2MASS-transformed-to-IRSF one ${ }^{1}$. The metric defined by the above equation finds the IRSF star that is closest in both position and magnitude to the SPM4 one. This procedure should minimise false matches.

Having constructed this SPM4 $\times$ IRSF catalog, one per Magellanic Cloud, we proceeded to cross-match the corresponding Be star candidates with them, this time using only the sky coordinates to find their match: for each Be star candidate, we found the closest SPM4×IRSF source within 3".

During the matching process in the LMC, we found twenty six cases where we had two Be star candidates matched within 3" with one SPM4xIRSF star. Among these multiple matches, the largest difference in colors B-V and V-I was about 0.2 magnitudes and generally smaller than 0.05 mags. Not having other information to decide, we chose the closest in angular separation as the most probable true match. We finally obtained 1188 Be star candidates with SPM4 proper motions and IRSF JHKs magnitudes.

During the matching process in the SMC, we found nine

1 Within the SPM4 LMC catalog, a few hundreds of stars did not have 2MASS JHK but instead had a value of 0 magnitudes in each band. For these SPM 4 stars, we took $\Delta \mathrm{J}=0$ by default, but chose a more restricted $\Delta \theta<2$ ". We then checked for repeated matches between this subset of SPM4 stars and the subset with 2MASS JHKs measured, and found eleven cases where two different SPM4 stars were matched to the same IRSF source, in each case one match had 2MASS JHKs and the other had 0 values, and in these few cases, we always chose the one with measured 2MASS JHKs. 


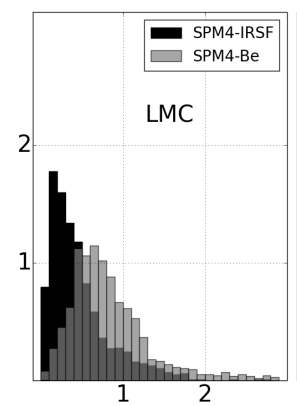

$\Delta \theta$ (arcsec)

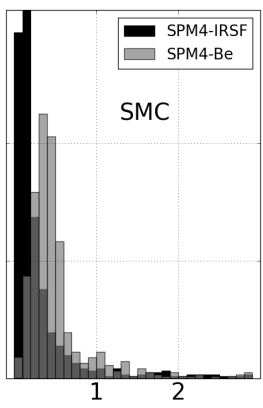

$\Delta \theta(\operatorname{arcsec})$

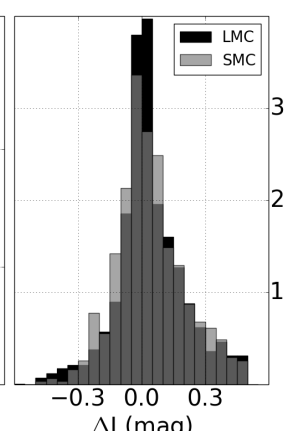

$\Delta \mathrm{J}(\mathrm{mag})$
Figure 1. Histograms of $\Delta \theta$ and $\Delta \mathrm{J}$ for Be star candidates in the LMC and SMC. The angular separation between the Be star candidate OGLE-II sky position and the one obtained from SPM4 is also plotted in grey in the first two panels. Notice how SPM4 and IRSF have a better agreement than SPM4 and OGLE-II.

cases where we had two Be star candidates matched within 3" with one SPM4xIRSF star, and in one case we had one Be star candidate matched within 3" with two SPM4xIRSF stars. Among these multiple matches, the largest difference in colors (B-V and V-I for the first nine cases, and magnitude $\mathrm{J}$ for the last one), was as observed in the LMC, and not having other information to decide, we again chose the closest in angular separation as the most probable true match. We finally obtained 619 Be star candidates with SPM4 proper motions and IRSF JHKs magnitudes.

Figure 1 shows the histograms of $\Delta \theta$ and $\Delta \mathrm{J}$ for each of these final matches catalogs, labeled as SPM4-IRSF. In the first two panels, we also include a histrogram of the sky angular separation in arcseconds between the SPM4 sky coordinate and the one listed by Sabogal et al. (2005) and Mennickent et al. (2002) (taken from OGLE-II), for the LMC and SMC, respectively, labeled as SPM4-Be. Table A1 (see Appendix) shows a few lines of the final data table for the LMC sample; the SMC table has the same structure. Sky equatorial coordinates and proper motions with their corresponding errors come from SPM4, BVI photometry comes originally from OGLE-II and JHKs photometry comes from IRSF, the OGLE-II ID as well as the Type of each Be star candidate comes from Sabogal et al. (2005) and Mennickent et al. (2002), for the LMC and SMC, respectively. Additional information regarding the quality of the proper motion (columns $\mathrm{m}, \mathrm{n}$ and i from SPM4), as well as the SPM4 ID are included, to identify a few stars in SPM4 whose proper motions are of lower quality due to the fact that the 2nd-epoch position was not measured neither in SPM plates $(n=0)$ nor CCDs $(i=0)$ but was taken from an input catalog. Such few stars are labeled as false SPM4 stars, as their proper motions are not measured exclusively with SPM4 data, although their first epoch material is from SPM4 ( $m$ indicates the number of 1st-epoch SPM4 plates used for each star, and for all SPM4 stars, always m>0.).

\section{SAMPLE SELECTION FROM COLOR-COLOR DIAGRAMS}

After close examination of various color-color and colormagnitude diagrams that could be done with the collected
Table 1. Photometry cuts that define samples A, B and C within each Magellanic Cloud. The number of so called false SPM4 stars within each sample is written in parenthesis.

\begin{tabular}{clcc}
\hline Sample & Definition & LMC & SMC \\
\hline A & V-I $\leq 0.6$ and $\mathrm{V}-\mathrm{I}>0.59(\mathrm{~V}-\mathrm{J})-0.3$ & $864(15)$ & $544(4)$ \\
B & $\mathrm{V}-\mathrm{I}>0.6$ and $\mathrm{V}-\mathrm{I}>0.59(\mathrm{~V}-\mathrm{J})-0.3$ & $216(3)$ & $35(1)$ \\
C & $\mathrm{V}-\mathrm{I} \leq 0.59(\mathrm{~V}-\mathrm{J})-0.3$ & $108(31)$ & $40(0)$ \\
\hline Total & & $1188(49)$ & $619(5)$ \\
\hline
\end{tabular}

BVIJHKs photometry, we were able to identify three mutually excluding samples, labeled A, B and C within each Magellanic Cloud, that had a distinctive clustering in all colours. Figures 2 (LMC) and 3 (SMC) show the results obtained in this regard. For both Clouds, these samples were defined with the same cuts in photometry, as described in Table 1. The number of false SPM4 stars within each sample is shown in parenthesis.

Such selection was motivated by the results obtained by Paul et al. (2012), where a sample of enigmatic candidates were obtained in the LMC Be star candidates, although not in the SMC, described as Type-4 stars with different optical and NIR properties. These stars "do not have NIR excess, show large reddening, but are not located in regions with high reddening. The reddening corrected magnitudes make them the brightest and most massive stars in the sample. Detailed spectroscopic studies are needed to understand these enigmatic candidates" (Paul et al. 2012). Within our study, such subgroup correspond to sample B within each cloud, and as expected, in the SMC such sample is much less populated. Our figures 2 and 3 replicate figures 3 and 4 from Paul et al. (2012), although no extinction correction was included, as it was deemed to small to have any significant effect in our investigation .

The histograms of $\Delta \theta$ shown in the lower right panel of figures 2 and 3 reveal that samples $C$ within each Magellanic Cloud are in fact mismatches between the Be star candidates and the SPM $4 \times$ IRSF catalog. Stars in samples $C$ have quite faint $\mathrm{V}$ magnitudes and J-H and H-Ks colors typical of GKM giants, as seen in the upper right panels of figures 2 and 3. Therefore, samples $\mathrm{C}$ within each Magellanic Cloud are discarded at this point from any further consideration, and only samples A and B are analysed in the following sections.

\section{PROPER MOTION RESULTS}

Figure 4 shows the vector points diagrams for samples A and $\mathrm{B}$ within each of the Clouds. In both cases, although especially for the LMC, it is evident that sample B is dominated by stars with large enough proper motions that place many of its stars within the Milky Way. This settles the nature of the originally thought as a new subclass of Be star candidates in the LMC suggested by Paul et al. (2012), as simply stars located within the Milky Way foreground, having noticeable larger values of $\mu_{\delta}$ than those expected for stars in the LMC. In the SMC, we also found that many stars in sample B show systematic larger values, this time easily visible in $\mu_{\alpha} \cos (\delta)$, when compared to the bluer sam- 


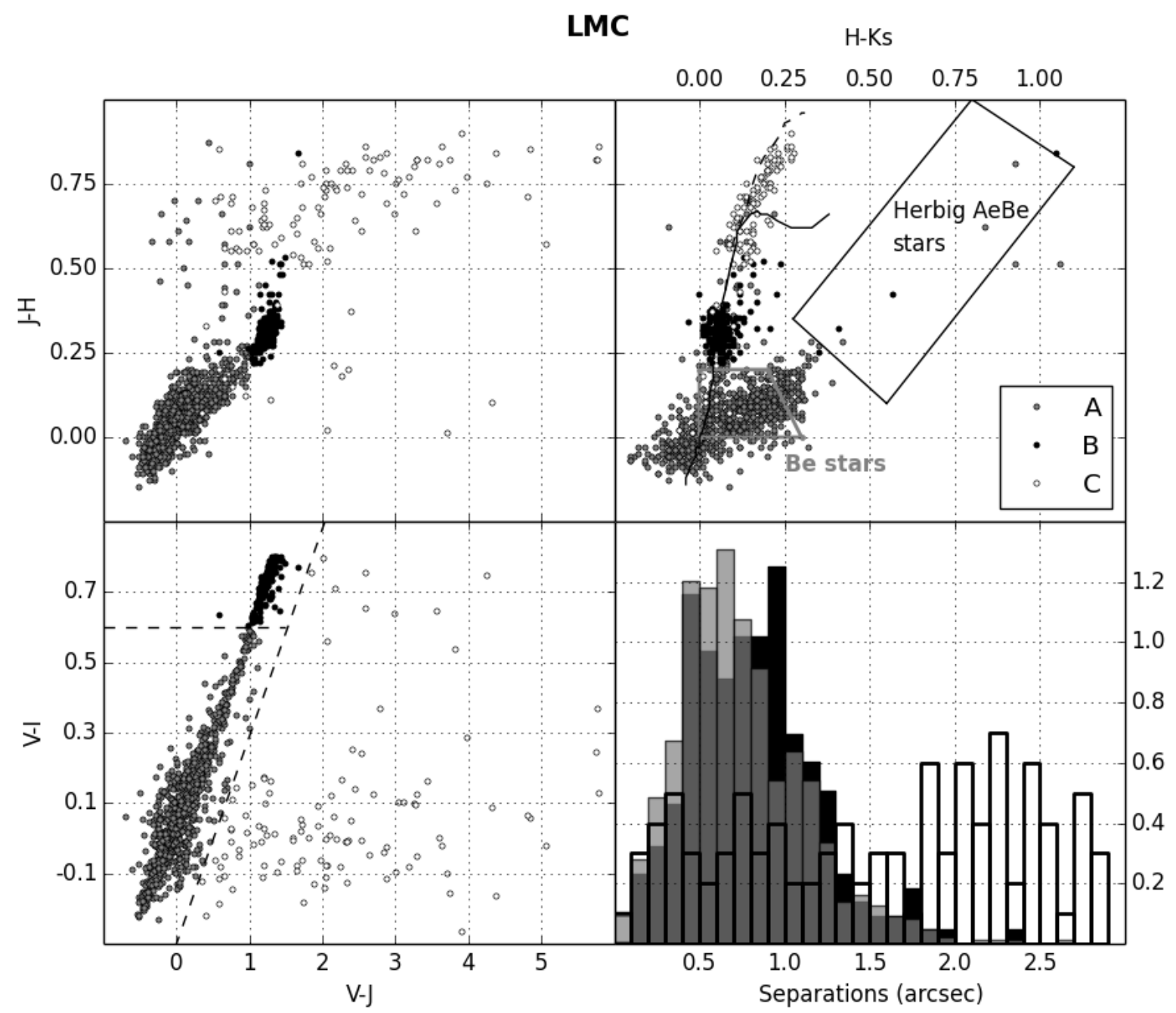

Figure 2. Color-color diagrams for the LMC Be star candidates with proper motions in SPM4 and histogram of angular separation between their OGLE-II and SPM4 sky positions, for samples A (gray), B (black), and C (white) within. The dashed straight lines in the lower left panel mark the photometry cuts that defined the samples. The solid and dashed curves in the upper right panel are the tracks of main sequence and giants stars, respectively, from Allen (2000). The grey polygon marks the location of Be stars from Dougherty et al. (1994) and the black polygon marks the location of Herbig Ae/Be stars from Hernández et al. (2005).

ple A. SMC's sample B is of a much smaller size than LMC's sample B.

This result is further confirmed when comparing the histograms of proper motions for all these samples with ones obtained from the SPM4 $\times$ IRSF catalog, using the photometric selection proposed by Nikolaev \& Weinberg (2000) based on JHKs magnitudes. Figure 5 shows the histograms of $\mu_{\delta}$ and $\mu_{\alpha} \cos (\delta)$ for the LMC and SMC, respectively, for their corresponding samples A and B, and for two samples chosen within each Magellanic Cloud, and named NW-A and NW-B respectively, defined as described in Table 2. Samples NW-A are dominated by stars in the Magellanic Clouds of spectral type and luminosity class O3-O5 V and B-A I-II, while samples NW-B are dominated by Milky Way disk stars of spectral type and luminosity class F-K V. It is important to notice that to select these NW-samples, we used IRSF JHKs magnitudes. It is visible that samples A and NW-A follow similar kinematics, and so do samples B and NW-B, within each Magellanic Cloud. This similarity refers mainly to central tendency values, that is that the mean values of samples A and NW-A are close to each other, and so are in samples B and NW-B, although regarding dispersion, each pair of samples also bear resemblance. Table 3 contains the number of data points, mean and unbiased standard deviation of $\mu_{\delta}$ for LMC, and $\mu_{\alpha} \cos (\delta)$ for the SMC, for their corresponding samples.

In any case, the main point of this investigation is to prove that samples B do not follow the kinematics expected for the Magellanic Clouds, that is that samples B proper motion distribution is noticeably different from their corresponding samples NW-A. Two-samples Welch's T-tests were performed on each possible pair of samples to test the null hypothesis that the means of the compared samples are the same. The obtained p-values are in Table 4 and indicate that 


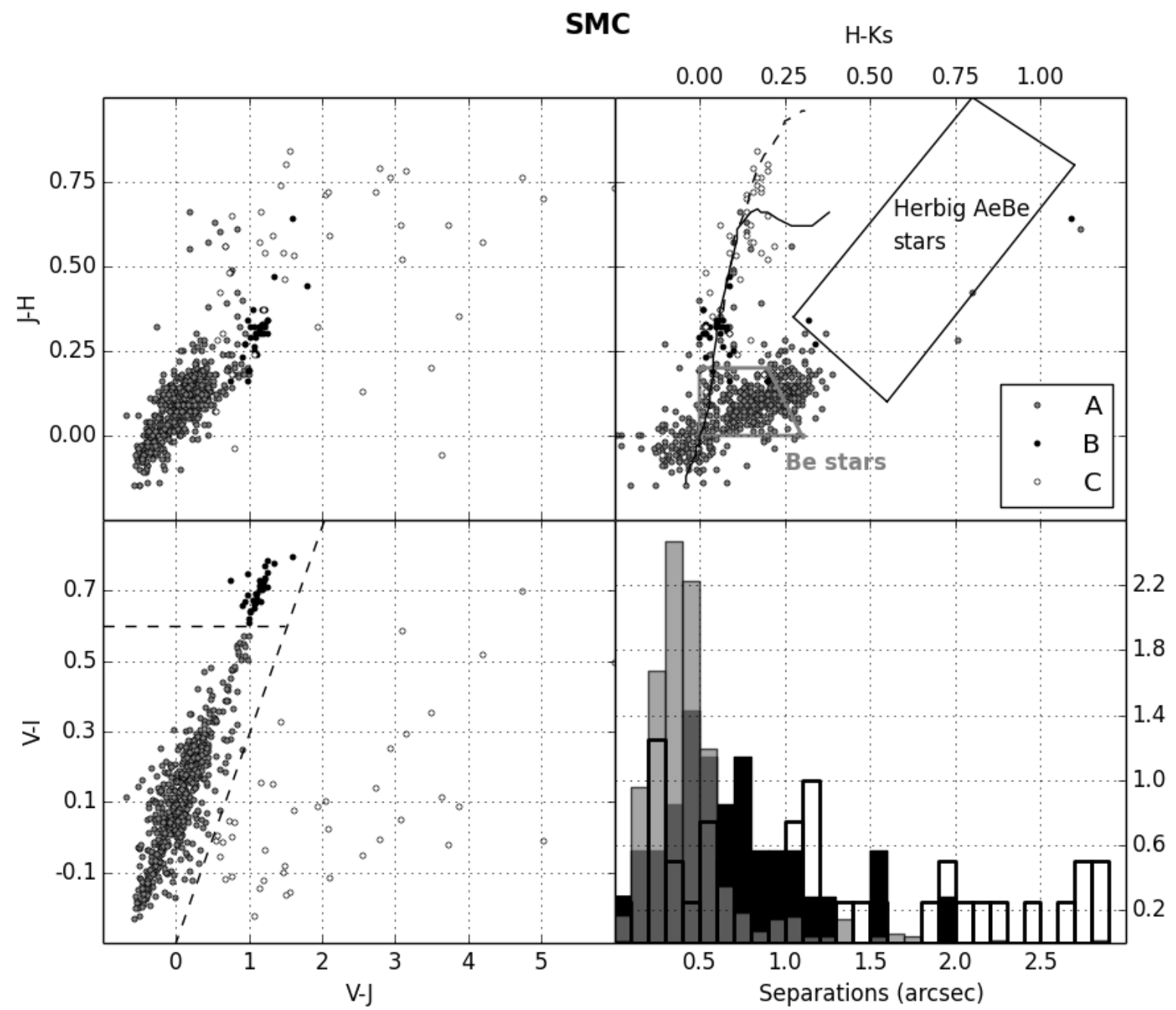

Figure 3. Same than Figure 2, but for the SMC.

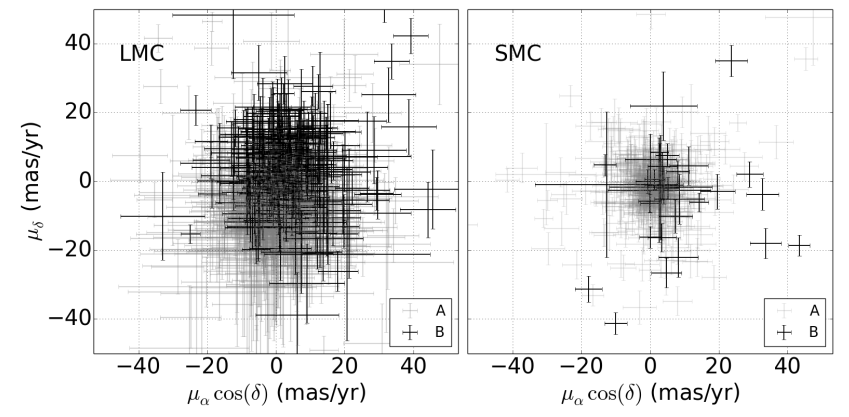

Figure 4. Vector-point diagrams for samples A and B within the LMC (left) and SMC (right). In each case, stars in sample B have proper motions too large to belong to the Magellanic Clouds and are therefore more probable members of the Milky Way.

the null hypothesis should be clearly rejected when comparing samples B vs. NW-A, and A vs. NW-B ones. These tests also show that the null hypothesis should not be indis- putably rejected in the other cases, that is A vs. NW-A and $B$ vs. NW-B, except on the LMC A vs. NW-A case, where it is visible that some difference exists in the means. Still in this case, we notice that their distributions overlap well enough to substantiate the idea that $\mathrm{A}$ and $\mathrm{NW}-\mathrm{A}$ share a similar kinematics, i.e. that many of the Be star candidates in the LMC sample A are indeed located in the LMC. It should be taken into account that SPM4 proper motion errors do affect the performance of these tests, and contamination from other populations or the presence of systematic effects in the SPM4 proper motions should be considered (See section 5 for an analysis of this last point).

\subsection{Cross-examination with some measured radial velocities}

In Paul et al. (2012), radial velocities and spectral types were determined for 102 Be star candidates drawn from Mennickent et al. (2002) and Sabogal et al. (2005), 39 and 63 stars in the LMC and SMC, respectively. In the SMC list, the star 
Table 2. Photometry cuts that define samples NW-A and NWB within each Magellanic Cloud, following Nikolaev \& Weinberg (2000) recipes for the LMC. For the SMC, the cut in Ks magnitude was extended 0.5 magnitudes fainter to account for its larger distance modulus, the color selection was kept the same.

\begin{tabular}{crll}
\hline Sample & \multicolumn{3}{c}{ Photometric cut } \\
\hline LMC NW-A & Ks $<14.75$ & and & J-Ks $<0.2$ \\
LMC NW-B & $\mathrm{Ks}<13.5$ & and & $0.2<\mathrm{J}-\mathrm{Ks}<0.5$ \\
\hline SMC NW-A & $\mathrm{Ks}<15.25$ & and & $\mathrm{J}-\mathrm{Ks}<0.2$ \\
SMC NW-B & $\mathrm{Ks}<14$ & and & $0.2<\mathrm{J}-\mathrm{Ks}<0.5$ \\
\hline
\end{tabular}

Table 3. Basic statistics for samples A, NW-A, B and NW-B in the LMC and SMC. SD stands for unbiased standard deviation.

\begin{tabular}{crrr}
\hline Sample & Size & Mean & SD \\
\hline \multicolumn{2}{c}{ LMC } & \multicolumn{2}{c}{$\mu_{\delta}$} \\
\hline A & 864 & -5.64 & 11.57 \\
NW-A & 3621 & -3.12 & 11.41 \\
B & 216 & 4.99 & 12.63 \\
NW-B & 4838 & 3.86 & 13.24 \\
\hline \multicolumn{2}{c}{ SMC } & \multicolumn{2}{c}{$\mu_{\alpha} \cos (\delta)$} \\
\hline A & 544 & 1.44 & 13.49 \\
NW-A & 1202 & 1.18 & 11.04 \\
B & 35 & 10.06 & 18.45 \\
NW-B & 1397 & 8.29 & 14.07 \\
\hline
\end{tabular}

Table 4. Welch's T-test results (p-values) for all possible comparisons of samples A, NW-A, B and NW-B in the LMC and SMC.

\begin{tabular}{ccccc}
\hline & \multicolumn{2}{c}{ LMC } & \multicolumn{2}{c}{ SMC } \\
& A & B & A & B \\
\hline NW-A & $1.01 \times 10^{-8}$ & $1.86 \times 10^{-17}$ & 0.91 & $5.38 \times 10^{-3}$ \\
NW-B & $1.85 \times 10^{-89}$ & 0.20 & $5.75 \times 10^{-11}$ & 0.18 \\
\hline
\end{tabular}

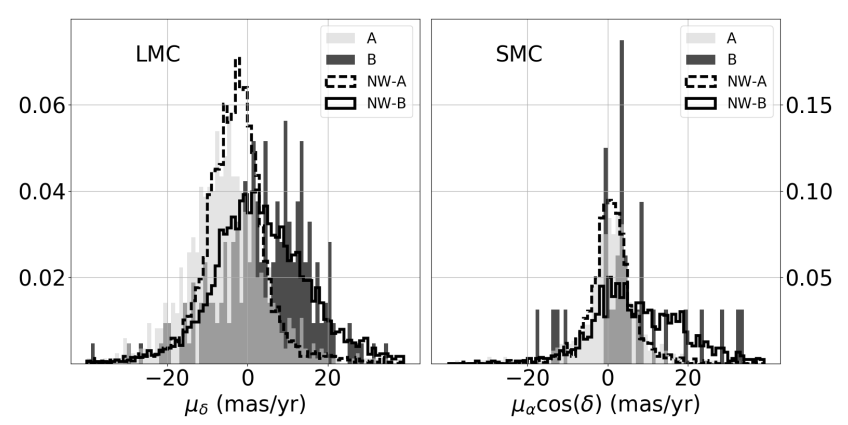

Figure 5. Histograms of $\mu_{\delta}$ and $\mu_{\alpha} \cos (\delta)$ for the LMC and SMC, respectively, for their corresponding samples $\mathrm{A}$ and $\mathrm{B}$, and for two samples chosen within each Cloud, and named NW-A and NW-B.

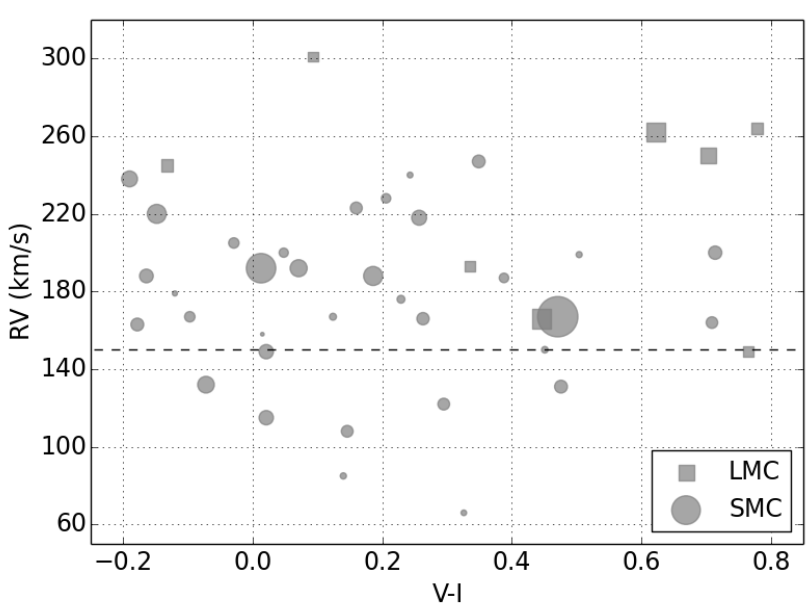

Figure 6. Radial velocity for Be stars with SPM4 proper motions versus their OGLE-II $V-I$ color, with a symbol sized by the total proper motion $\mu=\sqrt{\left(\mu_{\alpha} \cos (\delta)\right)^{2}+\left(\mu_{\delta}\right)^{2}}$.

with OGLE-II ID 005224.40-724038.6 appeared twice, with the same sky coordinates and photometry but with Types 1 and 2, and different radial velocities though still consistent with each other within errors $\left(V_{r}=149 \pm 30 \mathrm{~km} / \mathrm{s}\right.$ and $V_{r}=115 \pm 24 \mathrm{~km} / \mathrm{s}$, respectively). In fact this star was identified in our work and the Type-1 entry was taken for the match with SPM4, so in this case, for consistency we do the same again. From the LMC sample, 8 stars (out of 39) were identified in our work with proper motions in SPM4 and their data are displayed in Table A2. From the SMC sample, 33 stars (out of 63) were identified in our work with proper motions in SPM4 and the data for a few stars are shown in Table A3.

Figure 6 shows the radial velocity RV for these stars versus the V-I color ${ }^{2}$, with a symbol sized by the total proper motion $\mu=\sqrt{\left(\mu_{\alpha} \cos (\delta)\right)^{2}+\left(\mu_{\delta}\right)^{2}}$. Despite the fact that proper motion errors at the distance of the Magellanic Clouds represent a much larger error in $\mathrm{km} \mathrm{s}^{-1}$ as compared to the RV errors, it can be noticed how many of the SMC bluer stars have RV> $150 \mathrm{~km} \mathrm{~s}^{-1}$ and also the smaller proper motions, therefore all kinematical information is consistent with their membership to the SMC. For the LMC stars, with so few stars this plot is not as useful. In any case, this plot confirms that the bluer a variability-selected Be star candidate is towards the Magellanic Clouds, more probable it is to belong to these galaxies.

\section{DISCUSSION}

When finding trends of proper motions among colourselected samples, it is important to check that such behaviour is not the result of systematic errors within the catalog used, and therefore of non-cosmic origin, so to say.

In the Magellanic Clouds area, the SPM4 catalog has

2 Be star candidate with OGLE-II ID 004526.51-733014.2 in the $\mathrm{SMC}$ has $\mathrm{V}-\mathrm{I}=1.092$, out of the plot range and therefore is not shown in this figure. The same applies for Figure 7. 
measured proper motions, using observational materials of different kinds for their 2nd-epoch positions: photographic plates or CCDs (in a very few cases, these positions were directly taken from an input catalog, which can be Hipparcos, Tycho-2, UCAC2 or 2MASS, then $n=i=0$ ). Proper motions computed from 2nd-epoch CCD have errors about 2-3 times smaller than those from 2nd-epoch plates, and such errors are correlated with position in the sky, depending on the location of the plates. It can also happens that from one area in the sky with plates to another one with CCDs, an offset in the reference system takes place, artificially introducing trends in proper motions. Many astrometric catalogs suffer from these problems, but a thorough examination of the results generally reveals if such issues are present.

A quick examination of the proper motions in the Magellanic Clouds area within the SPM4 catalog, reveals some trends with $n$ that were inspected carefully to check if those could be behind the results described in the previous section. No trends in proper motion with $m$ or $i$ were observed, which in a catalogue like SPM4, are not unexpected.

Within the LMC SPM4 4 IRSF catalog, it is visible that stars with $n=0$ (no 2nd-epoch plates, therefore CCD positions if $i>0$ ) have an overall shift in their $\mu_{\delta}$ values towards positive values, as compared to the stars with $n>0$, whose global distribution looks more centered towards zero. Within the LMC Be sample, about $10 \%, 20 \%$ and $70 \%$ of the stars have $n=0, n=1$ and $n=2$, respectively. The proper motion trend in $\mu_{\delta}$ is the same for $n=1,2$ and as described in the previous section, and for $n=0$ is a bit offset but still sample $\mathrm{B}$ shows positive and larger values in $\mu_{\delta}$ as compared to sample A, as shown in Fig. 7, upper panel. Within samples $\mathrm{A}$ and $\mathrm{B}, n=1,2$ stars represent $73 \%$ and $83 \%$ of the stars, respectively. The majority of the $n=0$ stars are located towards the west end of the LMC Be stars in the sky. Within the SMC SPM4 $\times$ IRSF catalog, no visible trend in the proper motions with $n$ was visible. Within the SMC Be sample, about $80 \%$ of the stars have $n=0$, and the same proportion is seen within sample A and sample B. Figure 7 , lower planel, shows $\mu_{\alpha} \cos (\delta)$ vs. $V-I$ color-coded by $n$, which in this case ranges from 0 to 4 . We conclude that the observed trends in proper motions are a real feature of the samples chosen and not a bias caused by systematics in the SPM4 catalogue.

\section{CONCLUSIONS}

- A proper motion investigation for a sample of Be star candidates towards the Magellanic Clouds, using data from the SPM4 catalog has been done, which has allowed us to separate a Galactic foreground population from the Magellanic background one.

- The Galactic foreground population has redder colors in $\mathrm{B}-\mathrm{V}$ and $\mathrm{V}-\mathrm{I}$ and large proper motions visibly different from those expected for the Magellanic Clouds.

- In the LMC, this population is readily visible as a cluster in the J-H vs. J-Ks color-color diagram and had been described in the literature as an intriguing subgroup of stars within the LMC, that needed further spectroscopic analysis to clarify their nature.

- Our result proves that these stars are simply not in the Magellanic Clouds and confirms that the photometric

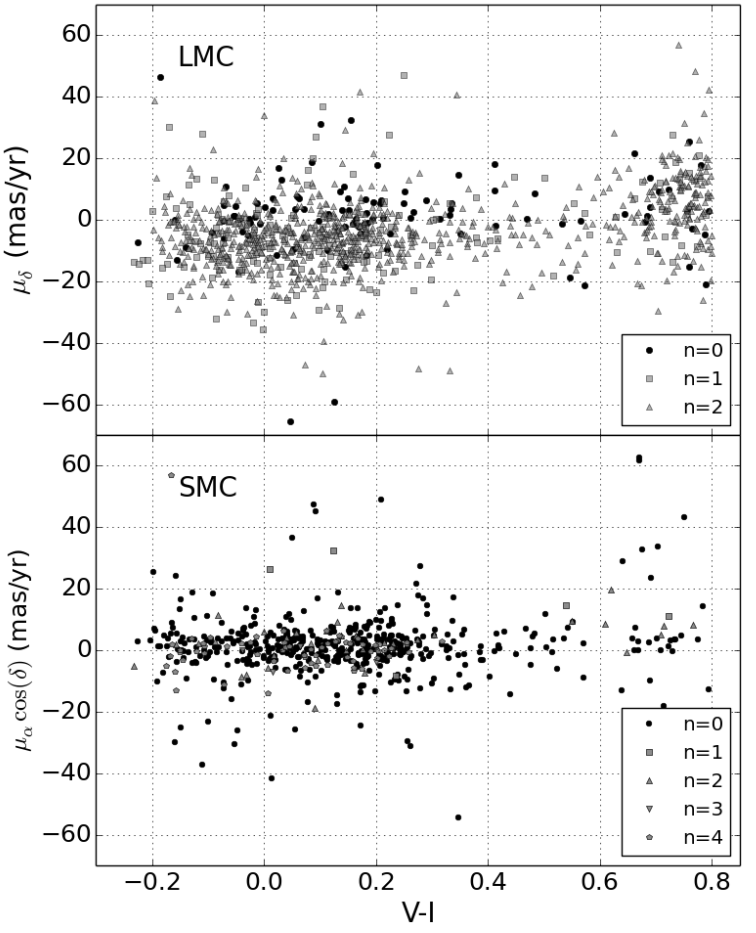

Figure 7. Proper motion vs. V-I, for different values of $n$.

variability method used by Mennickent et al. (2002) and Sabogal et al. (2005) to select Be star candidates, suffers from significant contamination towards redder B-V and V-I values.

\section{REFERENCES}

Allen C.W. 2000, in Cox A., ed., Allen's Astrophysical Quantities, 4th edition Springer-Verlag, New York

Collins, G. W. II. (1987) In IAU Colloq. 92: Physics of Be stars. p.3

Dougherty S. M., Waters L. B. F. M., Burki G., Cote J., Cramer N., van Kerkwijk M. H., Taylor A. R., 1994, å, 290, 609

Girard T. M. et al., 2011, AJ, 142, 15

Hernández J., Calvet N., Hartmann L., Briceño C., Sicilia-Aguilar A., Berlind P., 2005, AJ, 129, 856

Kato D. et al., 2007, PASJ, 59, 615

Mennickent R. E., Pietrzinsky G., Gieren W., Szewczyk O., 2002, A\&A, 393, 887

Mennickent R. E., Sabogal B., Granada A., Cidale L., 2009, PASP, 121, 125

Nikolaev S. and Weinberg, M. D. 2000, ApJ, 542, 804

Sabogal B. E., Mennickent R. E., Pietrzinsky G., Gieren W., 2005, MNRAS, 361, 1055

Paul K. T., Subramaniam A., Mathew B., Mennickent R. E., Sabogal B., 2012, MNRAS, 421, 3622

Rivinius T., Carciofi A. C., Martayan, C., 2013, A\&ARv, 21, 69 


\section{$8 \quad$ K. Vieira}

\section{APPENDIX A: TABLES}

A sample of Table A1, Table A2 and a sample of Table A3 are shown in the following page.

This paper has been typeset from a $\mathrm{T}_{\mathrm{E}} \mathrm{X} / \mathrm{L} \mathrm{LT}_{\mathrm{E}} \mathrm{X}$ file prepared by the author. 


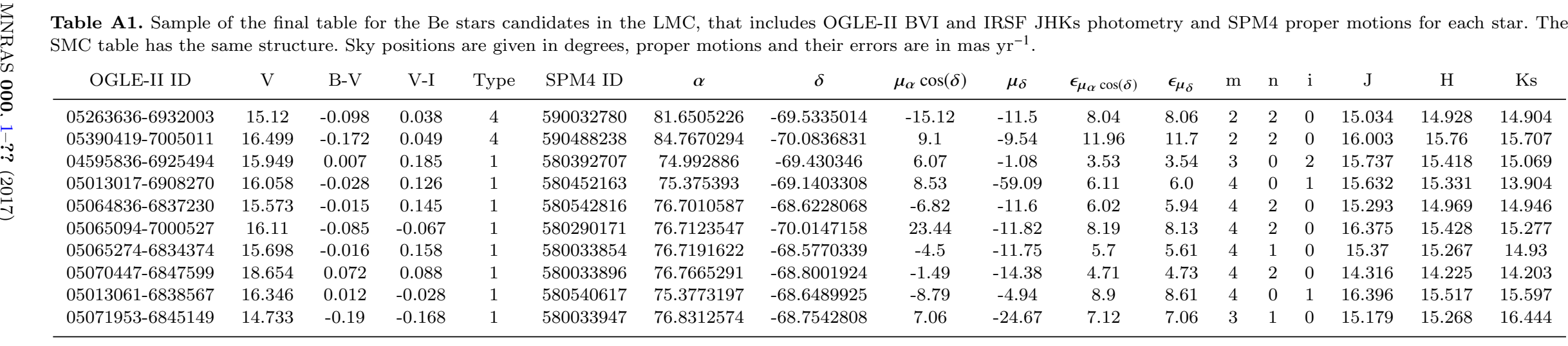

Table A2. Be stars candidates in the LMC that have full kinematic information, e.g. radial velocity and proper motions. Radial velocities and their errors, RV and e RV, are given in $\mathrm{km} \mathrm{s}^{-1}$, proper motions and their errors are in mas $\mathrm{yr}^{-1}$.

\begin{tabular}{cccccccccc} 
OGLE-II ID & Spectral Type & RV & $\mathrm{e}_{\mathrm{RV}}$ & $\mathrm{V}-\mathrm{I}$ & $\mathrm{SPM} 4 \mathrm{ID}$ & $\mu_{\alpha} \cos (\delta)$ & $\mu_{\delta}$ & $\epsilon_{\mu_{\alpha} \cos (\delta)}$ & $\epsilon_{\mu_{\delta}}$ \\
\hline $050052.36-685803.7$ & F9 & 149 & 2 & 0.765 & 580033194 & 4.62 & -2.98 & 2.19 & 2.24 \\
$050303.70-690615.0$ & A9-F0 & 193 & 33 & 0.335 & 580027763 & -1.84 & 5.28 & 2.39 & 2.44 \\
$051647.54-694415.2$ & F3 & 264 & 33 & 0.779 & 580023041 & 0.52 & -6.85 & 4.74 & 4.76 \\
$051744.42-692033.3$ & B8-A1 & 301 & 13 & 0.093 & 580029761 & 3.92 & -3.1 & 3.38 & 3.44 \\
$051947.82-693912.3$ & F6 & 166 & 43 & 0.446 & 580023530 & -11.34 & 13.93 & 4.98 & 5.04 \\
$052032.26-694224.2$ & F6 & 262 & 20 & 0.622 & 580346495 & 7.47 & -14.68 & 4.8 & 4.81 \\
$052258.47-692621.0$ & F6 & 250 & 99 & 0.703 & 580030795 & 4.67 & -10.82 & 4.87 & 4.89 \\
$052402.01-694920.5$ & B2-B3 & 245 & 22 & -0.132 & 580024625 & 5.89 & 3.35 & 4.4 & 4.47 \\
\hline
\end{tabular}

Table A3. Be stars candidates in the SMC that have full kinematic information, e.g. radial velocity and proper motions. Radial velocities and their errors, RV and e $\mathrm{RV}$, are given in $\mathrm{km} \mathrm{s}^{-1}$, proper motions and their errors are in mas $\mathrm{yr}^{-1}$.

\begin{tabular}{cccccccccc} 
OGLE-II ID & Spectral Type & RV & $\mathrm{e}_{\mathrm{RV}}$ & $\mathrm{V}-\mathrm{I}$ & $\mathrm{SPM} 4 \mathrm{ID}$ & $\mu_{\alpha} \cos (\delta)$ & $\mu_{\delta}$ & $\epsilon_{\mu_{\alpha} \cos (\delta)}$ & $\epsilon_{\mu_{\delta}}$ \\
\hline $005224.40-724038.6$ & B0-B3 & 149 & 30 & 0.021 & 290026279 & -5.77 & 8.01 & 2.85 & 2.94 \\
$004554.14-731404.3$ & A7-F0 & 164 & 37 & 0.709 & 280020464 & -0.06 & -6.46 & 2.63 & 2.71 \\
$004750.14-731316.4$ & B8 & 167 & 69 & 0.471 & 290213545 & -77.67 & -7.86 & 4.08 & 4.21 \\
$004921.41-725844.9$ & A7-F0 & 199 & 30 & 0.504 & 290025783 & 0.81 & -1.59 & 4.14 & 4.26 \\
$005025.64-725807.1$ & A7-F0 & 150 & 29 & 0.451 & 290025912 & -1.4 & -1.5 & 3.95 & 4.05 \\
$005043.44-732705.3$ & & 192 & 58 & 0.013 & 290190227 & -41.61 & 0.36 & 3.39 & 3.45 \\
$005359.22-723508.9$ & & 66 & 23 & 0.326 & 290026564 & -0.12 & -1.5 & 3.14 & 3.23 \\
$005745.25-723532.1$ & B2-B5 & 220 & 23 & -0.148 & 290027028 & 16.43 & 5.42 & 2.36 & 2.43 \\
$010000.78-725522.9$ & & 240 & 24 & 0.243 & 290027215 & 0.32 & -1.58 & 3.82 & 3.93 \\
$010043.94-722604.8$ & B0-B3 & 188 & 14 & -0.164 & 290032264 & 8.91 & -1.62 & 2.07 & 2.13 \\
\hline
\end{tabular}

